evaluated by RT-PCR and real-time RT-PCR. Proliferative responses and cell cycle analysis were detected by a CCK-8 assay and flow cytometry, respectively. ALP activity and ALP mass were detected by an ALP activity assay and ALP staining, respectively.

Results: In primary osteoblasts, only the IL-17 receptor was expressed. In C2C12, MC3T3-E1, and Saos-2 cells, the genes of IL-17, IL-22, and IL-23 receptors were not detectable. None of IL-17, IL-22, and IL-23 had an obvious effect on the proliferation of primary osteoblasts, but IL-17 exhibited an inhibitory effect on the gene expression of ALP, OCN, and Runx-2. The ALP activity and ALP mass of primary osteoblasts were downregulated by IL-17 treatment in a dose-dependent manner.

Conclusions: Primary osteoblasts constitutively express IL-17 receptors, but none of $\mathrm{C} 2 \mathrm{C} 12$ cells, MC3T3-E1 cells, and Saos-2 cells express any receptors for IL-17, IL-22, and IL-23. IL-17 inhibits BMP-2-induced osteoblast differentiation. Disclosure of Interest: None declared

DOI: 10.1136/annrheumdis-2018-eular.1659

\section{FRI0172 INFLAMMATION ON MRI OF THE SACROILIAC JOINTS IS HIGHLY PREDICTIVE OF STRUCTURAL DAMAGE IN AXIAL SPONDYLOARTHRITIS PATIENTS IN CLINICAL PRACTICE: DATA FROM THE ASAS AND DESIR COHORTS}

A. Sepriano ${ }^{1}$, S. Ramiro ${ }^{1}$, R. Landewée ${ }^{2}$, M. Dougados ${ }^{3}$, D. van der Heijde ${ }^{1}$, M. Rudwaleit ${ }^{4}{ }^{1}{ }^{1}$ Rheumatology, Leiden University Medical Centre, Leiden; ${ }^{2}$ Rheumatology, Amsterdam Rheumatology and Clinical Immunology Center (ARC), Amsterdam, Netherlands; ${ }^{3}$ Rheumatology, Hôpital Cochin, Hôpitaux de Paris, Paris, France; ${ }^{4}$ Rheumatology, Klinikum Bielefeld, Bielefeld, Germany

Background: The effect of MRI-detected inflammation on the development of radiographic damage at the sacroiliac joints (SIJ) level in patients (pts) with axial spondyloarthritis (axSpA) has been previously shown when images were scored by trained central readers ${ }^{1}$. Central reading decreases measurement error, but does not translate easily to what is usually done in clinical practice.

Objectives: To test the possible effect of MRI-SIJ inflammation on structural damage in X-SIJ, when both are assessed by local readers as in daily clinical practice.

Methods: Pts with axSpA (according to the treating rheumatologist) from both the ASAS and DESIR cohorts were included. MRI-SIJ and X-SIJ were obtained at baseline (BL), and at follow-up (ASAS: mean 4.4 years; DESIR: 5 years) and scored by local readers (rheumatologists/radiologists). Images were taken unblinded to other imaging information and clinical characteristics. Readers had the option to view the baseline image when scoring the follow-up image. Bone Marrow Oedema (BME) at MRI-SIJ was assessed either without a formal definition (ASAS-cohort) or according to the ASAS definition (DESIR-cohort) as present/absent. Structural damage in the X-SIJ was defined according to the $\mathrm{mNY}$ criteria. The\% of structural net progression (number of 'progressors' minus the number of 'regressors' divided by the total number of pts) was assessed in subgroups according to CRP and BME status at BL. The effect of BME on MRISIJ on X-SIJ damage was evaluated in logistic regression models adjusted for potential confounders selected a priori on clinical grounds (gender, HLA-B27, CRP, symptom duration, variables available in both cohorts).

Results: In total, 150 (ASAS-cohort) and 421 (DESIR-cohort) pts had complete 5 year X-SIJ data available. Remarkably, but not unexpectedly, the\% of 'improvements in X-SIJ' was impressive both in the ASAS- and DESIR-cohorts $(10 \%$ and $5.7 \%$ respectively), yielding a total\% of net progression that was higher in the former than in the latter $(14.7 \%$ and $5.9 \%)$. Net progression in X-SIJ ranged from $0.0 \%$ to $33 \%$ and from $0 \%$ to $17.4 \%$ according to the presence of objective signs of inflammation at BL in the ASAS- and DESIR-cohorts, respectively (figure 1). In the multivariable analysis, the presence of baseline BME at MRI-SIJ both in the ASAS (OR=3.2 [95\% Cl: 1.3-7.9]), and DESIR cohorts (OR=7.4 [95\% Cl: 4.312.7]) was highly predictive of X-SIJ structural progression at follow-up (table 1).

Abstract FRI0172 - Table 1. Effect of inflammation on MRI-SIJ at baseline on X-SIJ structural damage at follow-up

\begin{tabular}{lc}
\hline Predictor Outcome & $\mathrm{mNY}$ \\
& OR $(95 \% \mathrm{Cl})$ \\
\hline Sacroiliitis on MRI-SIJ (ASAS-cohort) & $3.2(1.3 ; 7.9)^{*}$ \\
Sacroiliitis on MRI-SIJ (DESIR-cohort) & $\mathbf{7 . 4 ( 4 . 3 ; 1 2 . 7 )}$ \\
\hline${ }^{*}$ Adjusted for gender, HLA-B27, CRP, symptom duration. &
\end{tabular}

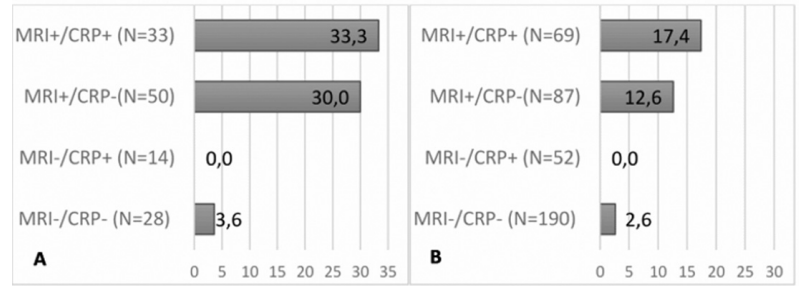

Abstract FRI0172 - Figure 1. Net progression from mNY-negative to mNY-positive according to baseline objective inflammatory markers in the ASAS (A) and DESIR (B) cohorts (ASAS: $\mathrm{N}=125 ; 25$ patients miss baseline MRI-SIJ; DESIR: $\mathrm{N}=398 ; 6$ patients miss baseline MRI-SIJ and 17 patients miss baseline CRP).

Conclusions: Our results, obtained in two independent cohorts, show that despite the expected increased 'noise' (measurement error) invoked by local reading, inflammation on $\mathrm{MRI-SIJ}$ still clearly predicts the later development of radiographic damage in axSpA.

\section{REFERENCE:}

[1] Dougados, et al. Ann Rheum Dis. 2017;76(11):1823-1828.

Disclosure of Interest: None declared

DOI: 10.1136/annrheumdis-2018-eular.2419

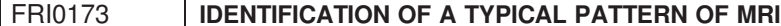 LESIONS OF SACROILIAC JOINTS IN PATIENTS WITH OSTEITIS CONDENSANS ILII AS COMPARED TO AXIAL SPONDYLOARTHRITIS}

D. Poddubnyy ${ }^{1,2}$, N. Gobejishvili ${ }^{1}$, T. Diekhoff ${ }^{1}$, H. Weineck ${ }^{1}$, M. Llop ${ }^{1}$, V. Rios Rodriguez ${ }^{1}$, J. Sieper ${ }^{1}$, K.-G. Hermann ${ }^{1}$. ${ }^{1}$ Charité UniversitäTsmedizin Berlin; ${ }^{2}$ German Rheumatism Research Centre, Berlin, Germany

Background: Osteitis condensans ilii $(\mathrm{OCI})$ is regarded as a non-inflammatory disorder that is believed to be induced, for example, by mechanical stress and mechanical instability of the sacroiliac joints (SIJ) related to pregnancy/delivery. $\mathrm{OCl}$ is being increasingly recognised as an important differential diagnosis for axial spondyloarthritis (axSpA), due to onset at young age, possible inflammatory character of back pain and recently described presence of subchondral bone marrow oedema on magnetic resonance imaging (MRI) of the SIJ. So far, no systematic comparison of MRI changes in the sacroiliac joints in patients with $\mathrm{OCl}$ and axSpA has been performed.

Objectives: To compare active and chronic inflammatory lesions of the SIJ as detected by MRI in patients with $\mathrm{OCI}$ and axSpA

Methods: Using medical database search we identified $n=103$ patients aged $\geq 18$ years who were diagnosed with $\mathrm{OCl}$ upon presentation with chronic back pain in the Early Spondyloarthritis Clinic of the rheumatology department in the Charité University Hospital between January 2010 and May 2015. These patients were contacted in order to obtain an informed consent and to complete a survey on the disease-related history. A total of 27 patients had evaluable MRIs of the SIJ in STIR and T1-weighted sequences, which were used for the current study. These patients were matched to 27 patients with definite axSpA according to the back pain duration. MRIs were scored according to the Berlin scoring system for osteitis, fatty degeneration, erosions, sclerosis and ankylosis independently by 3 trained and calibrated readers who were blinded for all clinical data including diagnosis. In addition, the preferential localization of lesions (ventral, mid, or dorsal part of the SIJ) was recorded.

Results: There were no differences either in the osteitis score or in the proportion of patients with presence of osteitis on MRI of the SIJ between OCI and axSpA patients (table 1). The fatty degeneration score was significantly lower in $\mathrm{OCl}$ as compared to axSpA, although the difference in the prevalence of the fatty lesions did not reach the level of statistical significance. There was a non-significant trend towards a higher sclerosis score in $\mathrm{OCl}$ patients. Importantly, there was a highly significant difference in the erosion score and in the prevalence of erosions: only 2 (7.4\%) OCI vs. 18 (66.7\%) axSpA patients had at least one erosion (table 1). Importantly, none of the $\mathrm{OCl}$ patients had high-grade ( $>5$ erosions) erosive changes. 